\title{
COMMUNICABLE DISEASES REPORT, NEW SOUTH WALES, FOR JANUARY AND FEBRUARY 2006
}

For updated information, including data and facts on specific diseases, visit www.health.nsw.gov.au and click on Infectious Diseases.

\section{TRENDS}

Tables 1 and 2 and Figure 1 show reports of communicable diseases received through to the end of February 2006 in NSW.

\section{ROSS RIVER VIRUS INFECTION ON THE RISE}

Ross River virus (RRV) is spread by mosquitoes that feed on infected animals and people. The virus then multiplies within the mosquito and is passed to other animals or people when the mosquito feeds again. Infections tend to peak in the summer and autumn months. The virus is not spread directly from one person to another.

Many people who are infected with the virus will never develop symptoms. Some people will have flu-like symptoms that include fever, chills, headache and aches and pains in their muscles and joints. Some joints can become swollen, and joint stiffness may be particularly noticeable in the morning. Sometimes a rash occurs on the body, arms or legs. The rash usually disappears after seven to 10 days. There is no specific treatment for Ross River virus infection.

In recent weeks NSW has experienced a rise in reports of RRV infection. There were 210 cases with a reported onset in February and 190 in January 2006, compared with 103 in December, 67 in November and 17 in October 2005 (see Figure 2). This compares with only 34 reported cases in January 2005. Cases have been largely reported from rural areas.

Prevention depends on avoiding being bitten by mosquitoes, especially in the summer and autumn months when infections peak. Advice includes:

- avoid being outside unprotected before dawn and after dusk

- when outside wear loose fitting, light coloured clothing that covers your arms and legs

- use an insect repellent that contains the chemical DEET (N, N-diethyl-m-toluamide) or picaridin

- fit fly screens to all windows, doors and chimneys and keep them in good repair

- use a knockdown insecticide (following the advice of the manufacturer) in bedrooms approximately half an hour before going to bed.

\section{LEGIONNAIRES' DISEASE CLUSTER}

Sydney West Public Health Unit staff reported a cluster of five cases of Legionnaires' disease with reported onset in January 2006, including four in the Blacktown area. The five cases were all men aged between 50 and 78 years. Legionella pneumophila serogroup 1 was cultured in sputum from one case. The others had positive urinary antigen tests. All cases were interviewed about exposures during their incubation periods (2-10 days before onset).

The investigation found that three of the five men had visited the Blacktown shopping area during their incubation periods. Common exposures in the Prospect and Seven Hills areas were also reported by two men. South Western Public Health Unit staff, assisted by Blacktown Council, reviewed the maintenance of cooling towers in these areas to ensure compliance with the NSW Public Health Act 1991 (for details of these requirements, see: www.health.nsw.gov. au/public-health/ehb/general/microbial/microbial.html). In addition, the public health unit staff contacted Emergency Departments and general practitioners in the area to advise them to report other possible cases. There is no evidence of ongoing risk.

\section{A CASE OF CUTANEOUS ANTHRAX}

In January, Greater Southern Public Health Unit staff reported a case of cutaneous anthrax in a man from the state's west. The man lived on a property in the 'anthrax belt' that runs down the west of NSW. Anthrax has survived for many decades as spores in the soil in this part of the country, and animals are occasionally diagnosed with anthrax, so farmers routinely vaccinate their animals to prevent disease. The man reported minor trauma to his arm around December 2005 while working on farm machinery. It was at this site that pimples developed and then eschars, which are black ulcers that are typical of anthrax. In late January 2006 anthrax was cultured from the patient's eschars. The patient was treated with penicillin and recovered. For more information see www.health.nsw.gov. au/infect/diseases.html and scroll down to 'Anthrax'.

\section{ENTERIC DISEASE}

Some types of Escherichia coli bacteria can produce toxins (Shiga toxins). These bacteria are called Shiga toxigenic $E$. coli (STEC) or verotoxigenic producing E. coli (VTEC). Various strains of VTEC (for example E. coli $\mathrm{O} 111$ and E. coli $\mathrm{O} 157$ ) have been responsible for outbreaks of serious human disease, including bloody diarrhoea that can sometimes be complicated by haemolytic uraemic syndrome (HUS), a severe condition characterised by kidney failure, bleeding and anaemia. VTEC infection may be acquired by eating contaminated food; commonly undercooked burgers, unwashed salad vegetables, and unpasteurised milk and milk products. ${ }^{1}$ Usually only one or two cases of VTEC or HUS are reported each year in NSW. 
From October 2005 to early February 2006, NSW Health received 12 notifications of cases of VTEC infection. Staff at one laboratory reported changing the laboratory's stool specimen screening method in 2005, which may account for some of the increase in notifications. The ages of the patients ranged from three to 85 years, and five were female.

Six reports of HUS were received in the same period in NSW. Consequently public health units were asked to undertake active surveillance for HUS cases by contacting renal physicians in their Areas to identify any unreported cases of HUS. As a result three additional cases of HUS were identified. Of all nine HUS cases, three were female, and the patients' ages ranged from one to 64 years. Three of the 9 HUS cases had associated VTEC infections, making a total of 15 VTEC cases for the period October 2005 to early February 2006.

All patients who had had HUS and VTEC were interviewed to identify any common exposures. None were identified.

Clinicians should report cases of HUS to their local public health unit on diagnosis, and test these cases for evidence of VTEC infection. To avoid infection it is important to:

- cook minced meat thoroughly, at least until any juices run clear

- wash salad vegetables thoroughly before eating

- avoid unpasteurised products.

VTEC may also be spread though contact with infected animals, especially cattle.

\section{Reference}

1. Heymann DL (ed). Control of Communicable Diseases Manual $18^{\text {th }}$ Ed. 2004: American Public Health Association, Washington.

\section{CRYPTOSPORIDIOSIS}

Reports of cryptosporidiosis remained elevated throughout summer. The high numbers were probably the result of an ongoing outbreak, and changes to procedures in some laboratories that have resulted in an increase in the routine testing of stools.

The outbreak seemed to begin in rural areas in spring 2005, when some cases were linked to contact with infected farm animals, or contact with other cases. Over summer, more than 50 per cent of cases reported swimming in pools during their incubation periods (in the previous two to 12 days), suggesting that swimming remains an important risk factor.
Public health units routinely investigate cases of cryptosporidiosis reported by laboratories, and where a cluster of cases linked to a pool is identified, public health unit staff contact the pool operators to ensure that the pool is maintained according to NSW Health guidelines (see: www. health.nsw.gov.au/public-health/ehb/water/recreational. html). People with diarrhoea should not enter swimming pools for at least a week after complete recovery.

\section{LISTERIOSIS}

Listeriosis is a rare but serious illness caused by eating food contaminated with the bacteria Listeria monocytogenes. The bacteria are common in soil and the environment. Most people do not get sick after exposure to Listeria, but some people, including pregnant women, unborn and newborn babies, older people and people with compromised immune systems, are at increased risk of illness.

In February, NSW Health was notified of a 34-year-old pregnant woman with listeriosis. The woman presented to hospital with a fever and back pain. She reported no knowledge of listeriosis and had been consuming foods considered to be of high risk throughout her pregnancy. Both mother and baby recovered.

The risk of exposure to Listeria can be reduced by a few food safety principles: wash your hands before preparing food, keep raw and cooked foods separate, store perishable foods in the fridge and consume as soon as possible, cook meats thoroughly and wash fruit and vegetables before eating.

Foods that are considered to be high risk and that should be avoided if you are at risk of listeriosis are:

- pre-packed salads and fruit

- pre-cooked diced chicken (as used on sandwiches)

- delicatessen sliced meats

- raw seafood

- $\quad$ soft cheeses (such as brie and camembert)

- $\quad$ sprouted seeds

- mushrooms.

Listeria survives at low temperatures so it is advisable to avoid foods that have been stored in the fridge for long periods of time.

For more information, see www.health.nsw.gov.au/publichealth/cdscu/facts/pdf/listeriosisfs.pdf. 
FIGURE 1

\section{REPORTS OF SELECTED COMMUNICABLE DISEASES, NSW, JAN 2000 TO FEB 2006, BY MONTH OF ONSET}

Preliminary data: case counts in recent months may increase because of reporting delays.

Laboratory-confirmed cases only, except for measles, meningococcal disease and pertussis $\mathrm{BFV}=$ Barmah Forest virus infections,

RRV = Ross River virus infections

Lab conf $=$ laboratory confirmed
Men $\mathrm{Gp} \mathrm{C}$ and $\mathrm{Gp} \mathrm{B}=$ meningococcal disease due to serogroup $C$ and serogroup $B$ infection, other/unk = other or unknown serogroups.

NB: multiple series in graphs are stacked, except gastroenteritis outbreaks.

NB: Outbreaks are more likely to be reported by nursing homes and hospitals than by other institutions

\begin{tabular}{|lr}
\hline \multicolumn{2}{|l|}{ NSW population } \\
Male & $50 \%$ \\
$<5$ yrs & $7 \%$ \\
$5-24$ yrs & $27 \%$ \\
$25-64$ yrs & $53 \%$ \\
$65+$ yrs & $13 \%$ \\
Rural & $46 \%$
\end{tabular}
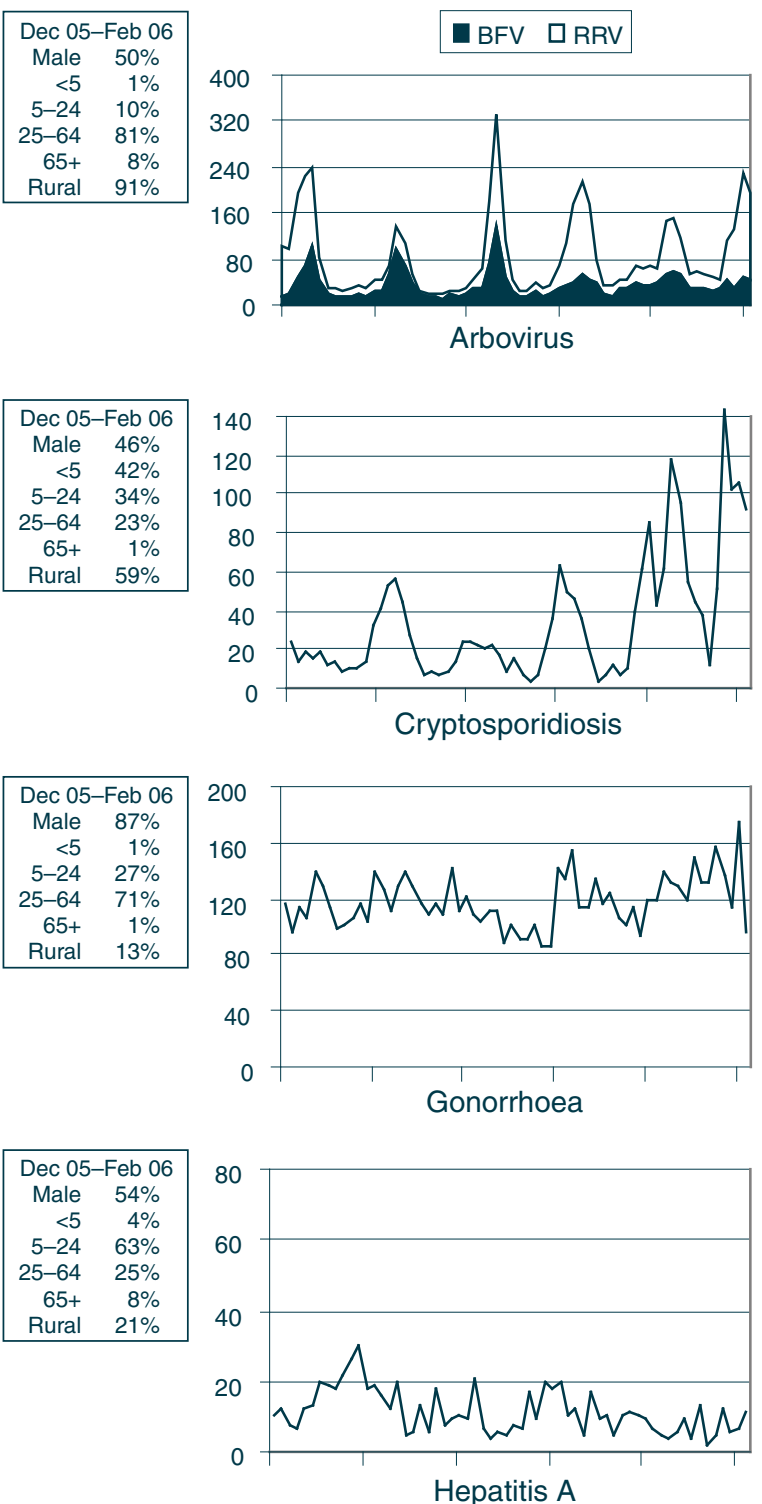

\begin{tabular}{|lr|}
\hline \multicolumn{2}{|c|}{ Dec 05-Feb 06} \\
All outbreaks & 12 \\
Nursing homes & 4 \\
Hospitals & 7 \\
Child care & 1 \\
Schools & 0 \\
Other & 0 \\
\hline
\end{tabular}

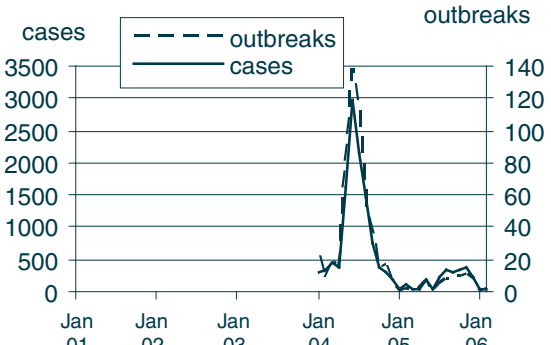

Gastroenteritis outbreaks in institutions

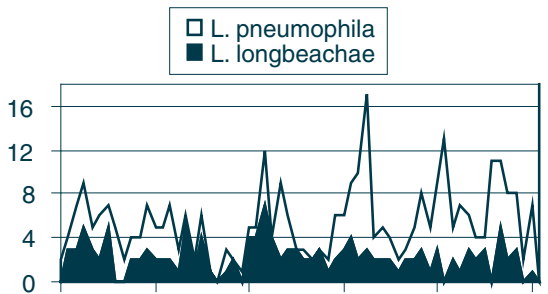

Dec 05-Feb 06 Male $80 \%$

$<5 \quad 0 \%$ 5-24 $0 \%$ $25-64 \quad 60 \%$

$\begin{array}{rr}65+ & 40 \% \\ \text { Rural } & 0 \%\end{array}$ Legionellosis

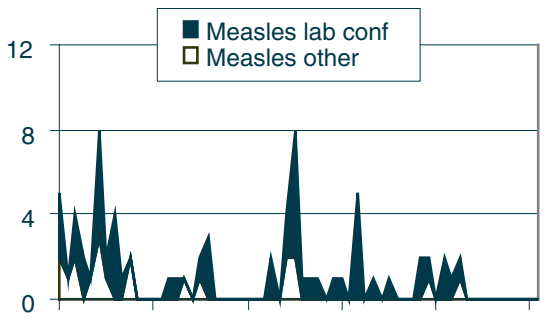

Dec 05-Feb 06 Male $0 \%$

$<5 \quad 0 \%$ 5-24 $0 \%$ 25-64 $0 \%$

$65+\quad 0 \%$
Rural

Rural $0 \%$

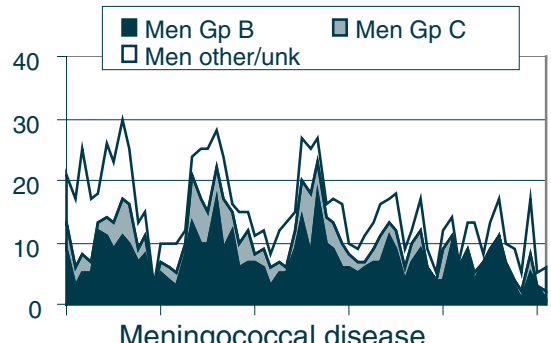

\begin{tabular}{|cc|}
\hline \multicolumn{2}{|c|}{ Dec 05-Feb 06 } \\
Male & $79 \%$ \\
$<5$ & $39 \%$ \\
$5-24$ & $39 \%$ \\
$25-64$ & $22 \%$ \\
$65+$ & $0 \%$ \\
Rural & $32 \%$ \\
\hline
\end{tabular}

Rural 32\%

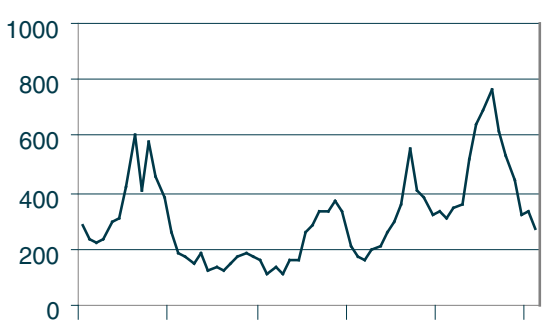

Dec 05-Feb 06

Male $\quad 40 \%$

$\begin{array}{rr}<5 & 2 \% \\ 5-24 & 11 \%\end{array}$

$25-64 \quad 71 \%$

$65+\quad 16 \%$

Rural $33 \%$

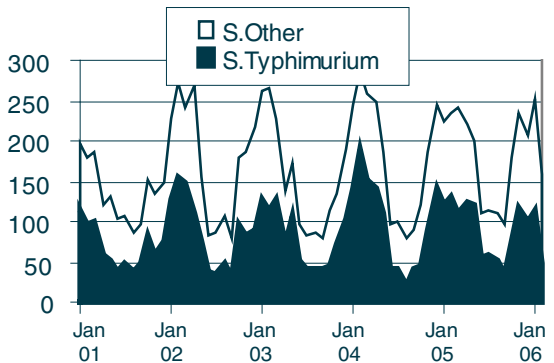

Salmonella infections

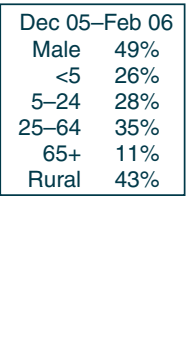

c $05-F e b$
ale

$<5 \quad 26 \%$

$35 \%$

$65+11 \%$

$43 \%$ 


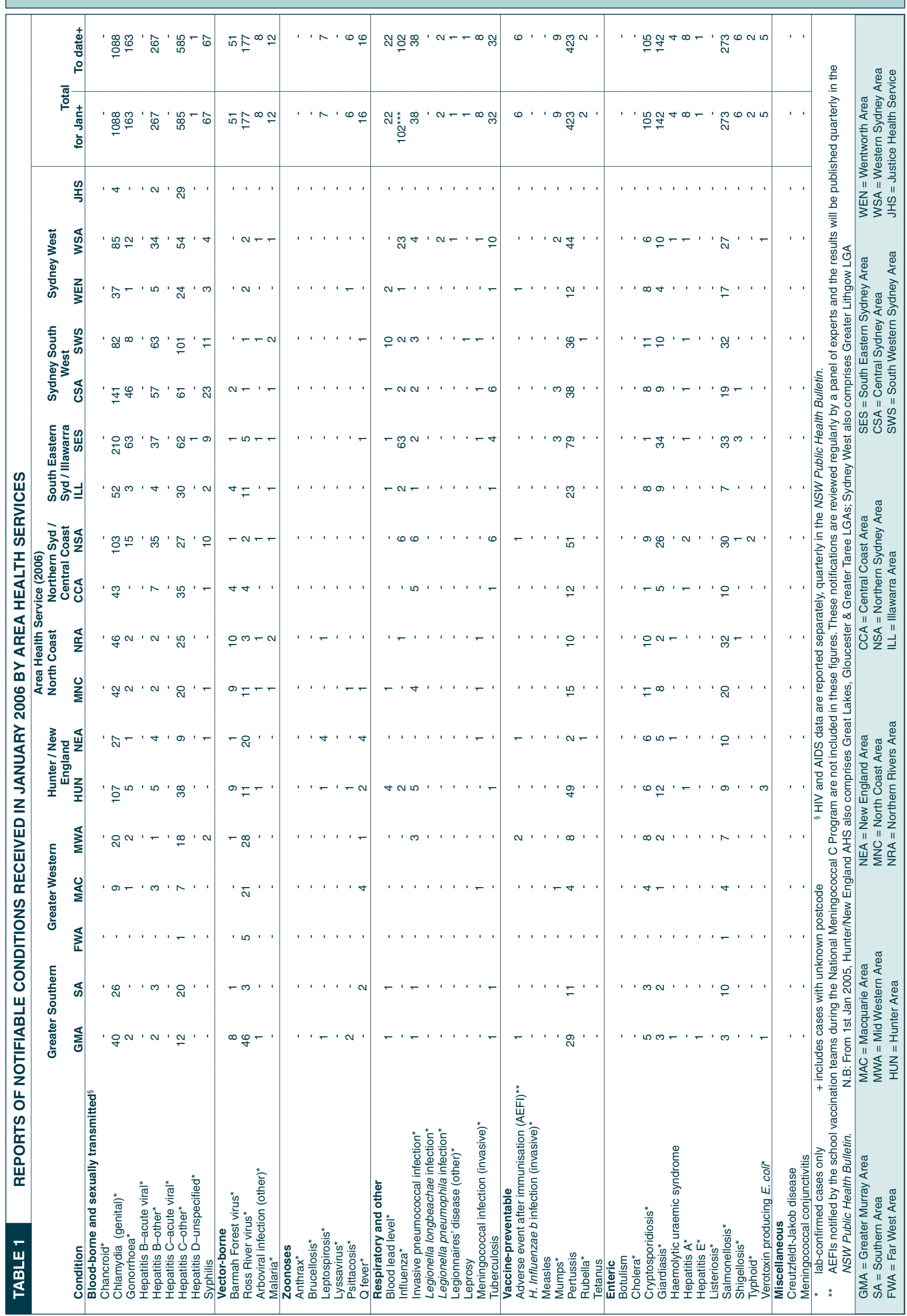




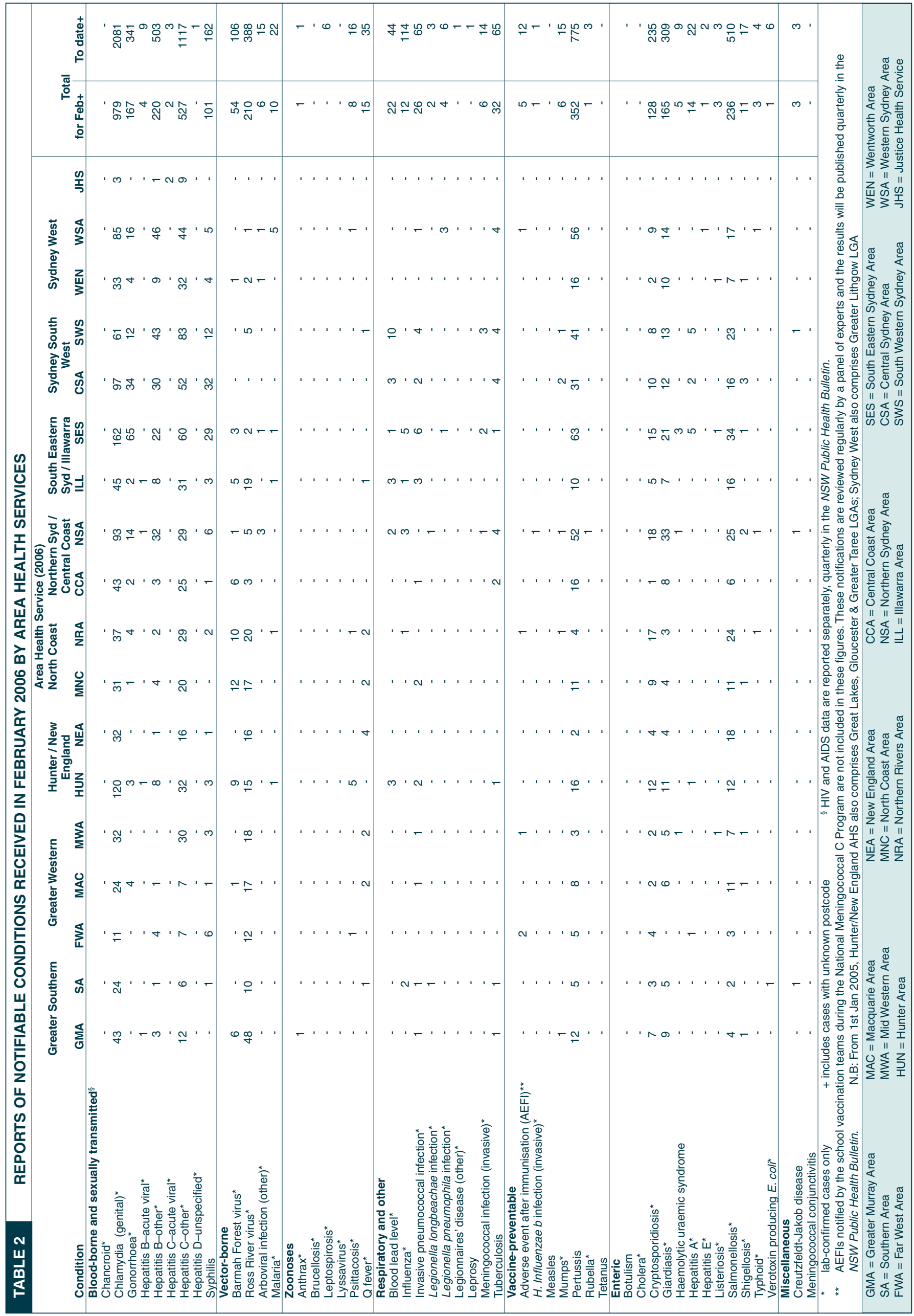

